\title{
Optical Properties of Copper Phthalocyanine(CuPc)Thin Films
}

\author{
M. M. El-Nahass, F.S. Bahabri* ands.R.Al-Harbi* \\ Faculty of Education, Ain Shams University, Cairo, Egypt. \\ * Faculty of Girls, Jeddah, Kingdom of Saudi Arabia,
}

\begin{abstract}
Vacuum sublimed thin films of copper phthalocyanine were prepared at room temperature. The optical properties of thin CuPc films have been investigated in the spectral range 200-2100 $\mathrm{nm}$. The refractive index $n$ as well as the absorption index $k$ were calculated before and after annealing. It is obvious that the refractive index showed an anomalous dispersion in the absorption region as well as normal dispersion in the transparent region. The analysis of the spectral behavior of the absorption coefficient, $\alpha$ in the absorption region revealed direct transitions. The analysis of $\alpha$ as well as the dielectric constants $\varepsilon_{1}$ and $\varepsilon_{2}$ reveals several absorption peaks at photon energies given in the text. The fundamental and the onset energy gaps could also be estimated as $2.74 \pm 0.02$ and $1.64 \pm 0.02 \mathrm{eV}$ respectively.
\end{abstract}




\section{Introduction}

Phthalocyanines are today clearly regarded as optical materials, thin applies to organic dye layers in general. The analysis of the optical film properties naturally enforces the application of the theoretical and experimental skills of thin film optics to the mentioned molecular system [1-3]. Another important fact emphasizes the significance of thin film spectroscopy in application to condensed molecular matter. The points is, that due to the weak Van der Waals interaction between molecules in compared with the free molecule [2-4]. These materials are generally p-type semiconductors and have the advantage of being sufficiently stable towards chemical and thermal treatment. They can easily be sublimed and resulting in high purity thin films without decomposition.

In the present work, thin films of copper phthalocyanine have been fabricated by thermal evaporation onto quartz substrates. Optical constants and oscillating parameters have been determined. Moreover the visible and near ultraviolet absorption spectra have been measured. The fundamental and the onset energy gaps could also be estimated and discussed.

\section{Experimental Details}

The copper phthalocyanine $(\mathrm{CuPc})$ powder used in this study is obtained from Kodak, U K (99.97\%). Thin CuPc films of different thicknesses were prepared by conventional thermal evaporation technique, using a high vacuum coating unit (Edwards type E 306 A, England). The films are deposited onto optically flat quartz substrates. Since the state of the substrate surface greatly affects the growth of the deposited film, therefore cleaning the surface of substrate is very important. The films were deposited from a quartz crucible source heated by a tungsten coil in a vacuum of $10^{-4}$ $\mathrm{Pa}$ during deposition, the temperature of substrates were kept at room temperature. The deposition rate was controlled at $2.5 \mathrm{nms}-1$ using a quartz crystal thickness monitor (Model FTM6, Edwards Co., England).

The transmittance $T(\lambda)$ and the reflectance $R(\lambda)$ of the films were measured at normal incidence in the spectral range $200-2500 \mathrm{~nm}$ using a double - beam spectrophotometer (JACO) model V-570 UV- VIS- NIR).

The absolute values of the measured transmittance and reflectance after correction of the absorbance and reflectance of the substrate are given by $[8,9]$

$$
\mathrm{T}=(1-\mathrm{Rq})
$$


where $I_{\mathrm{ft}}$ and $\mathrm{I}_{\mathrm{q}}$ are the intensities of light passing through the film-quartz system and that through the reference quartz, respectively, and $\mathrm{R}_{\mathrm{q}}$ the reflectance of quartz substrate, and

$$
\mathrm{R}=\mathrm{R}_{\mathrm{m}}\left[1+\left(1-\mathrm{R}_{\mathrm{q}}\right)^{2}\right]-\mathrm{T}^{2} \mathrm{R}_{\mathrm{q}}
$$

where $I_{m}$ is the intensity of light reflected from the reference mirror, $I_{f r}$ is the intensity of light reflected from the sample reaching the detector and $R_{m}$ the mirror reflectance.

To calculate the refractive index $\mathrm{n}$ and the absorption index $\mathrm{k}$ of the films, a computer program comprising a modified search technique [8] based on minimizing $(\Delta \mathrm{R})^{2}$ and $\left.\Delta \mathrm{T}\right)^{2}$, simultaneously was used, where

$$
\begin{aligned}
& \left.(\Delta \mathrm{R})^{2}=\mid \mathrm{R}_{(\mathrm{n}, \mathrm{k})}-\mathrm{R}_{\exp }\right)\left.\right|^{2} \\
& (\Delta \mathrm{T})^{2}=\left|\mathrm{T}_{(\mathrm{n}, \mathrm{k})}-\mathrm{T}_{\text {exp }}\right|^{2}
\end{aligned}
$$

where, $T_{\exp }$ and $R_{\exp }$ are the corrected values of $T$ and $R$ respectively, and $\mathrm{R}_{(\mathrm{n}, \mathrm{k})}$ and $\mathrm{T}(\mathrm{n}, \mathrm{k})$ are the calculated values of $\mathrm{T}$ and $\mathrm{R}$, using the Murmann's exact equations $[8,9]$.

\section{Results and Discussion}

The spectral distribution of $T(\lambda)$ and $R(\lambda)$ measured at normal incidence in the wavelength range 200-2100 nm for as deposited CuPc films in the thicknesses range $16.602 \mathrm{~nm}$ were shown in Fig. (1a). Measurements were also performed for the same samples after being annealed at $473 \mathrm{~K}$ under vacuum of $10^{-1} \mathrm{~Pa}$ for two hours and represented in Fig. (2b). It could be noted that at longer wavelengths $(\lambda>900 \mathrm{~nm})$ all films become transparent and no light is scattered or absorbed as $\mathrm{R}+\mathrm{T}=1$ i.e. nonabsorbent region. The inequality $(\mathrm{R}+\mathrm{T})<1$ at shorter wavelengths $(\lambda<900 \mathrm{~nm})$ due to existence of absorption (absorbing region). After the determination of the film thickness, the bivariance search technique have been used $[8,9]$ to determine the real and imaginary parts of the complex refractive index from the absolute values of transmittance and reflectance of films, using Murmann's exact formulas as mentioned above. Taking into account, the experimental error in measuring the film thickness to be $\pm 2 \%$ and in $T$ and $\mathrm{R}$ to be $\pm 1 \%$, the error in the calculated values of $\mathrm{n}$ and $\mathrm{k}$ was estimated to be $3 \%$ and $2.5 \%$, respectively. The optical constants were found to be independent of the film thickness for the studied films. As can be seen in Fig. (2) there are a distinct characterized peaks in the spectral distribution of absorbance of $\mathrm{CuPc}$ thin films before and after annealing. As observed, there is no effect of annealing temperature on the behavior of absorbance. 

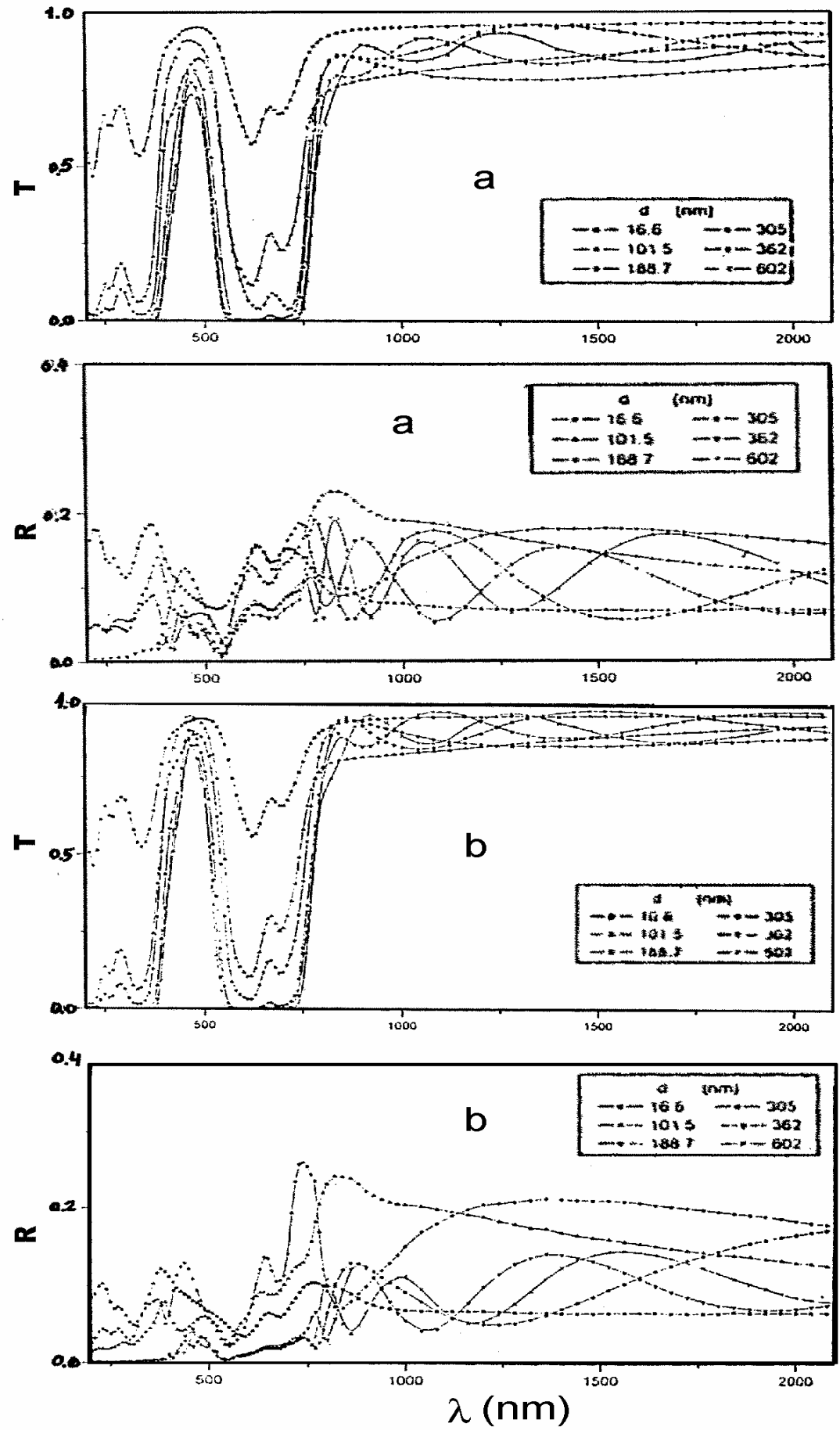

Fig. (1): The spectral distribution of $\mathrm{T}(\lambda)$ and $\mathrm{R}(\lambda)$ for : a) as deposited $\mathrm{CuPc}$ thin films and b) annealed $\mathrm{CuPc}$ thin films. 

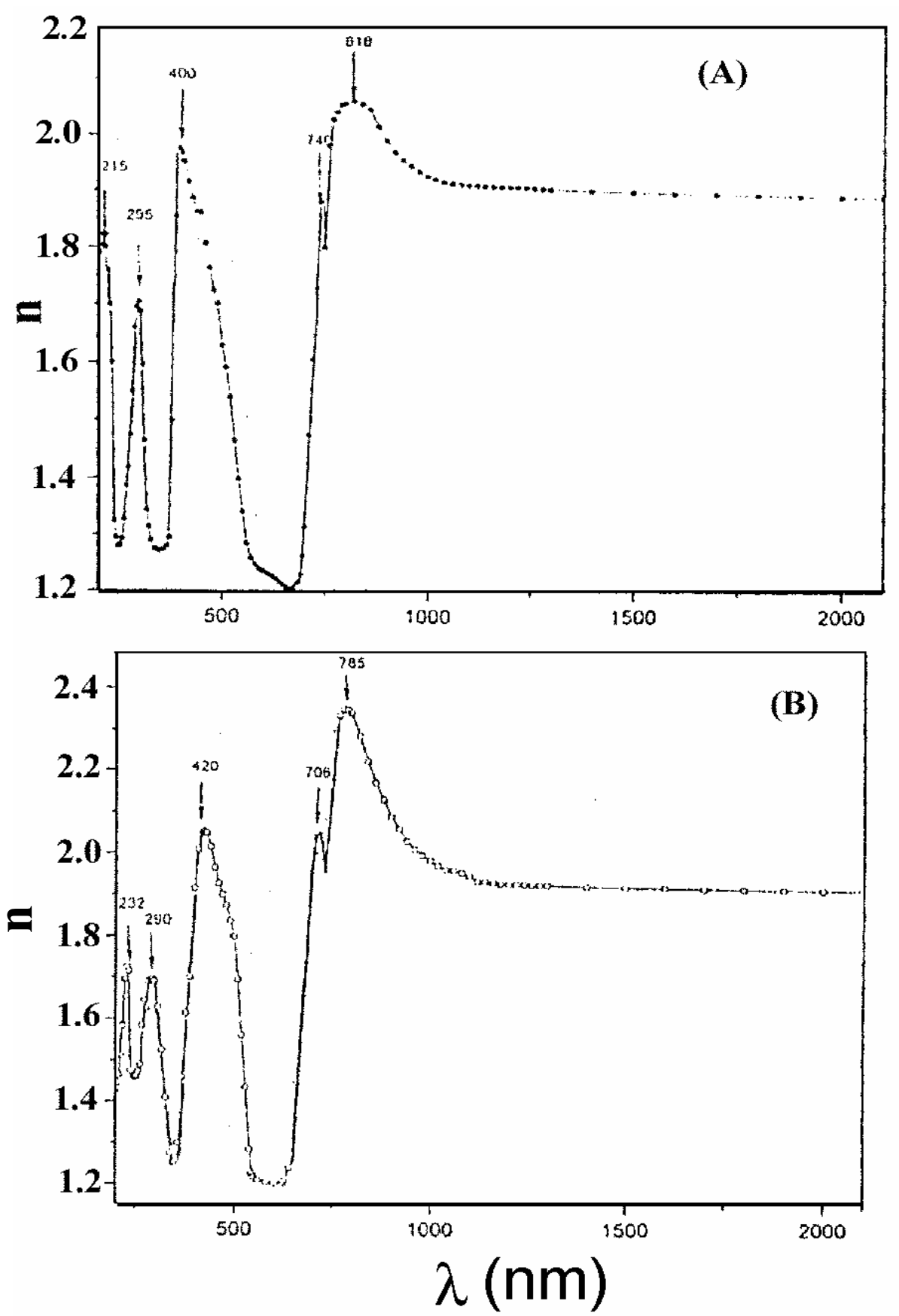

Fig. (2): The dispersion curve of refractive index $n(\lambda)$ for: a) As deposited CuPc thin films and $b$ ) Annealed $\mathrm{CuPc}$ thin films 
Fig. (2) shows the dispersion of the refractive index $n(\lambda)$, in the wavelength range $200-2100 \mathrm{~nm}$ for as-deposited $\mathrm{CuPc}$ films and after annealing. The observed values represent the mean values determined from films of different thicknesses.
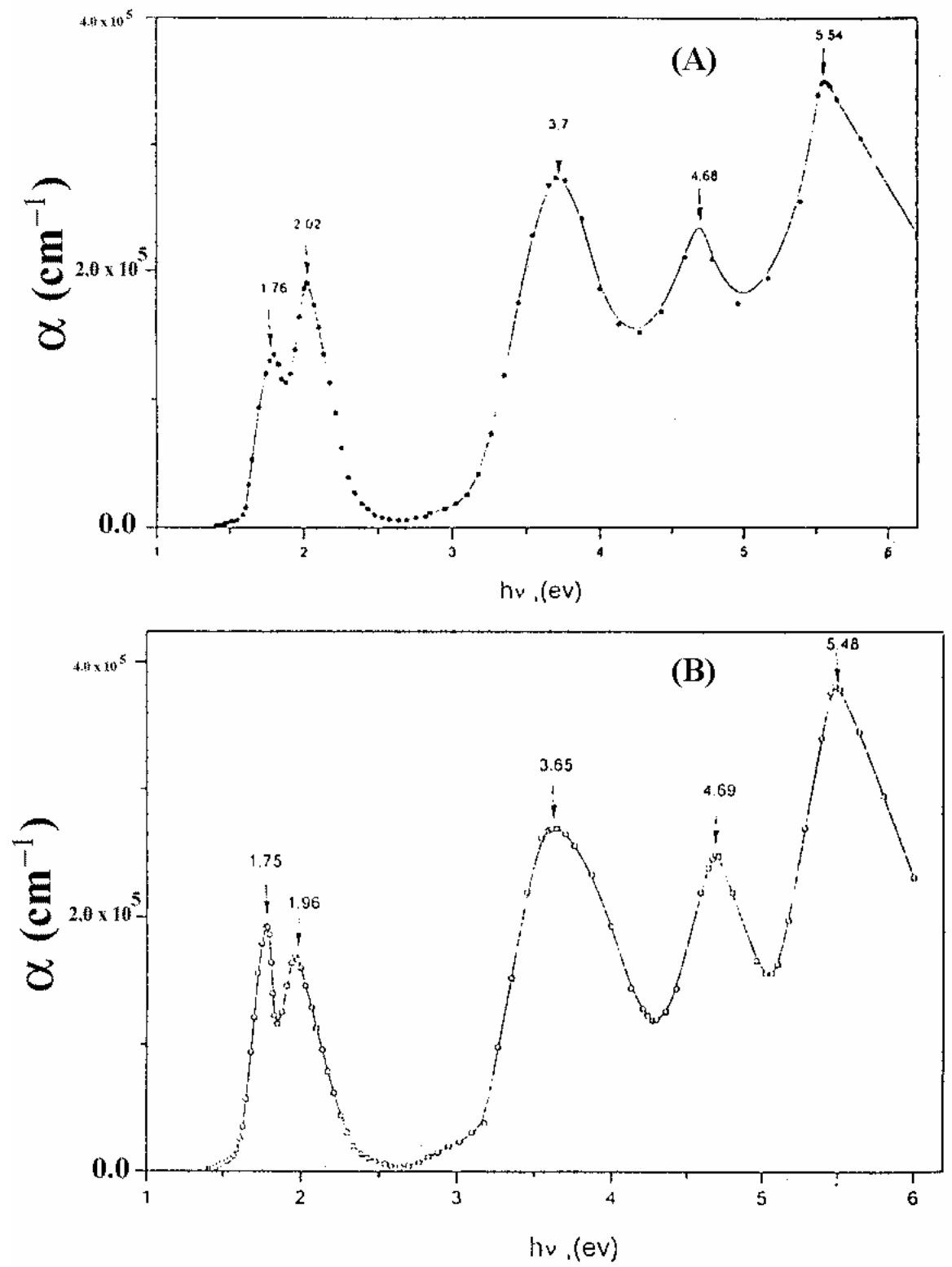

Fig. (3) The spectral behavior of the absorption coefficient a for A - As deposited $\mathrm{CuPc}$ thin films

B - Annealed CuPc thin films 
The spectral distribution of the absorption coefficient $\alpha=4 \pi \mathrm{k} / \lambda$ for the investigated $\mathrm{CuPc}$ films either as deposited or after being annealed is shown in Fig. (3). The distinct characterized peaks in the visible region have generally been interpreted in terms of $\pi-\pi^{*}$ excitation between bonding and antibonding molecular orbitals [10].

The high energy peak of the Q-band hand has been assigned assigned to the first $\pi-\pi^{*}$ transition on the phthalocyanine macrocycle [11]. The low energy peak of the Q-band has been variously explained as a second $\pi-\pi^{*}$ transition [11]. The present observation of similar structure of CuPc before and after annealing on the visible and Soret bands is taken as supporting evidence for explanation of structure in terms of a molecular vibrations [11]. The $\mathrm{N}$ peak of the Soret band indicates the presence of $\mathrm{d}$ band associated with the central metal atom. It is thought that $\pi$-d transitions are involved $\mathrm{CuPc}$ has partially occupied $\mathrm{d}$ band. Support in this explanation by Edwards and Gouterman [12] pointed out that transitions involving $d$ electrons of the central metal atom are provided. The absorption bands in the region of 4.5 to $5.7 \mathrm{eV}$ are due to $\mathrm{d}-\pi^{*}$ transitions [11], which implies a broader $\mathrm{d}$ band. Similar behavior of the absorption spectra are obtained by some workers $[11,13]$ for metal-derivative phthalocyanines.

To obtain information about direct or indirect inter-band transitions, the fundamental absorption edge data could be analyzed within the frame work of one electron theory of Bardeen et al. [14]. This theory has been used to analyze the absorption edge data of molecular solids such as phthalocyanine derivatives [15]. The absorption $\left(\alpha \geq 10^{4} \mathrm{~cm}^{-1}\right)$ is related to direct band transitions [16]. The variation in absorption coefficient with photon energy for direct band-to-band transitions is of the form

$$
\alpha=\alpha_{\mathrm{o}}(\mathrm{h} v-\mathrm{E})^{\mathrm{r}}
$$

where $\mathrm{E}$ is the energy gap. The value of $\mathrm{r}$ is $1 / 2$ for allowed direct transitions and $3 / 2$ for forbidden direct transitions. The direct allowed band gap was determined by plotting $\alpha^{2}$ as function of photon energy hv as shown in Fig. 4 and a satisfactory fit was obtained, showing the existence of a direct allowed gap at $2.74 \pm 0.01 \mathrm{eV}$ for films before and after annealing. These values of the energy gap result in the intense band called the Soret band and can be interpreted as a maximum in refractive index because the absorption index at that photon energy is quite small [16]. This energy gap can be attributed to the intense absorption in the red region as mentioned before [16] for $\mathrm{CuPc}$, $\mathrm{PtPc}$, and PbPc. The photon energy dependence of $\alpha^{2}$ for $\mathrm{CuPc}$ films before and after annealing is shown in Fig. (4), which found to be $1.64 \pm 0.02 \mathrm{eV}$. 

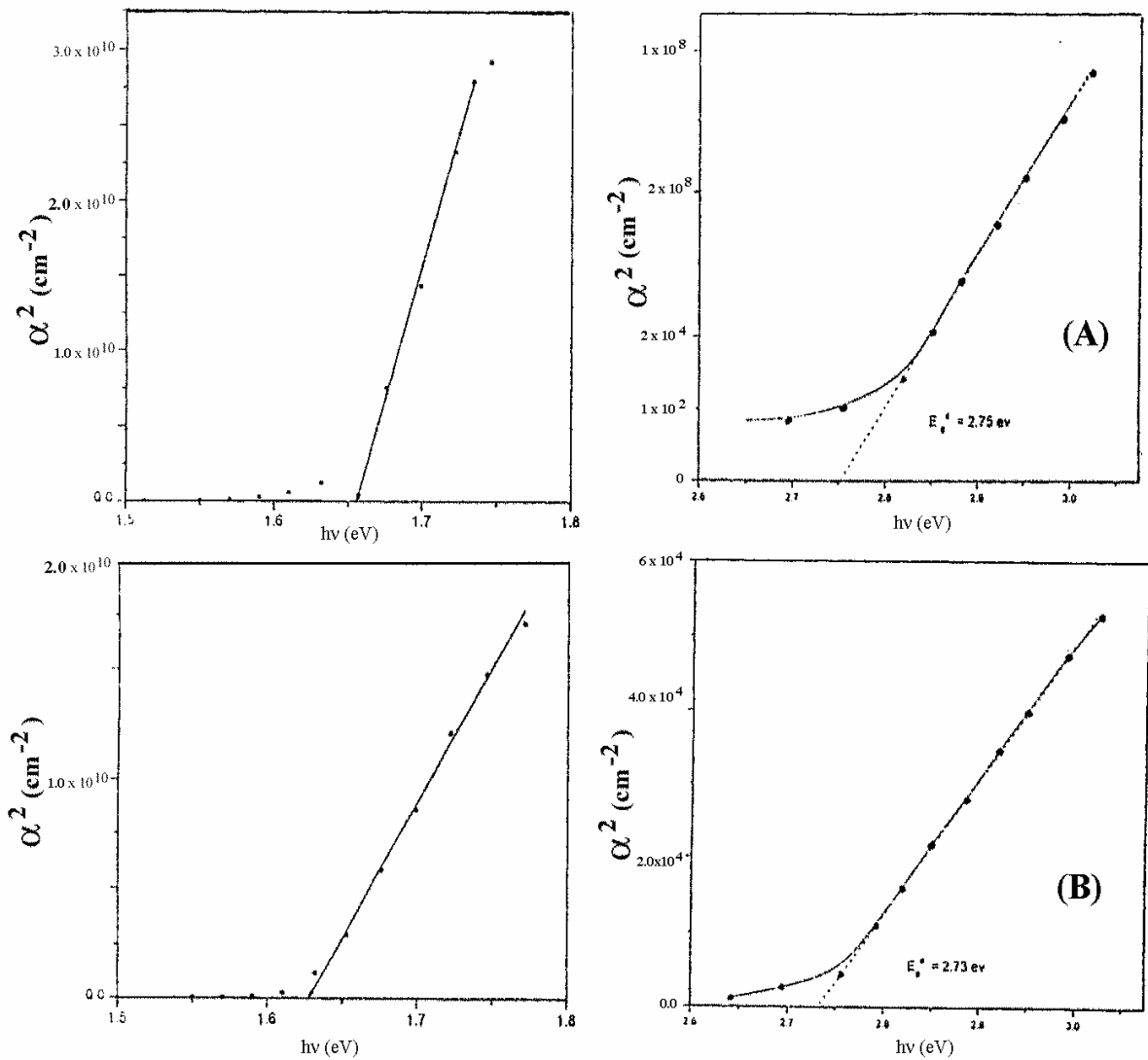

Fig. (4): The photon energy dependence of $\alpha .{ }^{2}$ for: a) as deposited CuPc thin films and b) Annealed CuPc thin films.

\section{Conclusions}

The optical properties of $\mathrm{CuPc}$ thin films before and after annealing have been studied in the spectral range $200-2100 \mathrm{~nm}$. The stability in the peak positions in transmission and reflection spectra in absorbing region showed the stability of the structure of $\mathrm{CuPc}$. The refractive index as well as the absorption index are practically independent on the film thickness. The spectral distribution of $\mathrm{k}$ of the investigated $\mathrm{CuPc}$ films characterized by a distinct peaks in visible region have generally been interpreted in terms of $\pi$ $\pi^{*}$ excitation. The absorption spectra occure in the high energy region of the Soret band (the $\mathrm{N}$ peak) indicate the presence of $\mathrm{d}$ band associated with the central metal atom. It is thought that $\pi$-d transitions are involved because $\mathrm{CuPc}$ has partially occupied $\mathrm{d}$ band. The fundamental energy gap result in 
the intense band called the Soret band can be interpreted as a maximum in refractive index because the absorption index at that photon energy is quite small. The onset energy gap indicates that the band-to-band absorption has taken place at lower energy. The results showed no effect of the annealing on the optical properties of $\mathrm{CuPc}$ films.

\section{References}

1. A. Mrwa, M. Friedrich, A. Hofman, Sensors and Actuators B24-25,596, (1995).

2. K. Nebesny, G.E. Collins, P.A. Lee, Chem, Mater. 3, 829, (1991).

3. M. Pope, C.E. Swenberg, "Electronic Process in Organic Crystals", Clarwndon Press, Oxford, (1992).

4. C.W. Tong, Appl. Phys. Lett. 48, 183 (1986).

5. C.C. Leznoff, A.B.P. Lever, Phthalocyanines Properties and Applications, 3, VCH, New York, (1993).

6. R.A. Collins, A.Belghachi, Mater. Lett. 8, 343 (1989).

7. Y.Machida, Y. Saito, a. Taomoto, Jpn. J. Appl. Phys. 28, 297 (1989).

8. M.M. El-Nahass, J. Mat. Sc. 27, 6597 (1992).

9. A.M.Bakry, A.H.El-Naggar, Thin Solid Films 360, 293 (2000).

10. S. Ambily, C.S. Menon, Thin Solid Films 347, 284, (1999).

11. A.T.Davidson, J. Chem. Phys. 77, 162 (1982).

12. L. Edwards, M. Gouterman, J. Mol. Spect. 33, 292 (1970).

13. A. Stendal, U.Beckers, S. Wilbrandt, O. Stenzel, V. Borezyskowski, J.Ph ye. B: At. Mol. Opt. Phys. 29, 2589 (1996).

14. J. Bardeen, F.J. Slatt, L. Hall, Photocnductivity Conf., 146, Wiley, New York, (1965).

15. S. Ambily, C.S. Menon, Sol. Stat. Commun. 94, 485 (1995).

16. A.K. Abass, Akrier, R.A. Collins, phys. stat. sol. (a)142, 435 (1994). 